\title{
Angina monocitica con sovrainfezione da Prevotella denticola: caso clinico
}

\author{
Maria Teresa Allù', Giovanni Provvidenza ${ }^{2}$ \\ ' Laboratorio Analisi Chimico - Cliniche e Microbiologiche Azienda Ospedaliera Ragusa \\ ${ }^{2}$ U.O. Otorinolaringoiatria Azienda Ospedaliera Ragusa
}

Key Words: Infectious mononucleosis, oral anaerobic bacteria, $\beta$-lactamase production

\section{SUMMARY}

\section{Monocytic angina with superinfection of Prevotella denticola: clinical case}

Monocytic angina is a clinical sindrome caused by Epstein-Barr virus characterized by fever, pharyngitis, exudative tonsillitis, swollen lymphoglands, splenomegaly and hepatomegaly. The inflamed pharynx and necrotic tonsils of infectious mononucleosis are subject to bacterial superinfection initially or during the course of the illness; the reduced $\mathrm{PO}_{2}$ tension and low oxidation-reduction potential that prevail in a vascular and necrotic tissues favour the growth of anaerobes.

In this article we reported the clinical case of a ten years old children, who presented fever and tonsillopharyngitis; he was treated with cefotaxime and piperacillin, he did not improve in health. He was admitted to hospital (Department of Otorhinolaryngology). The patient was treated with aminoglycoside (tobramycin), piperacillin and cortisone; the clinical situation deteriorated. Pus sample was collected from the tonsils and cultured. Isolated strain from culture anaerobic was identified biochemically (Rapid-ID $\left.{ }_{32} A N A\right)$. The microorganism isolated was: Prevotella denticola (oral anaerobic gram-negative rods); $\beta$-lactamase production was tested by using the chromogenic cephalosporin disk test. The susceptibility to antibiotics was performed according to NCCLS recommendations. Prevotella denticola ( $\beta$-lactamase production) was resistant to penicillin, cefoxitin, cefotetan, piperacillin, clindamycin and metronidazole it was susceptible to piperacillin-tazobactam, amoxicillin-clavulanate, ticarcillin-clavulanate, imipenem and chloramphenicol. Children was treated with piperacillin-tazobactam, with rapid symptomatic relief.

\section{INTRODUZIONE}

L'angina monocitica è un'angina ulcero-necrotica il cui agente eziologico è il virus di Epstein-Barr, che si replica principalmente nei linfociti $B$ ma può replicarsi nelle cellule epiteliali del faringe $\mathrm{e}$ del dotto parotideo. Il periodo di incubazione varia da 2 a 6 settimane; anche il decorso e la durata sono irregolari (1-4 settimane) (7). I sintomi soggettivi caratteristici dell'angina monocitica sono: febbre elevata, mialgia, astenia, faringodinia e odinofagia, scialorrea, poliadenite laterocervicale e retro-nucale ed epatosplenomegalia. L'aspetto tonsillare è caratterizzato dalla presenza di lesioni ulcero-necrotiche talvolta eritematopoltaceo. La diagnosi viene fatta sulla base del reperto clinico e dei dati di laboratorio: 1'emocromo evidenzia una leucocitosi con linfomonocitosi assoluta, risulta positiva la ricerca degli anticorpi contro gli antigeni precoci, del capside virale (VCA-IgM e VCA IgG) (2).

Prevotella denticola è un bacillo pleomorfo Gram-negativo, asporigeno e anaerobio obbligato. I microrganismi del genere Prevotella sono componenti della popolazione microbica orale e sono responsabili di infezioni della cavità orale, del tratto respiratorio superiore e purulente orofacciali. Gli anaerobi causano infezioni endogene e sono in grado di colonizzare i tessuti necrotici, danneggiati da traumi e da scarsa irrorazione sanguigna, dove è presente una ridotta $\mathrm{PO}_{2}$ ed un basso potenziale di ossido-riduzione, elementi favorevoli per la moltiplicazione degli anaerobi (5). Essi producono enzimi che giocano un ruolo fondamentale nell'instaurarsi dell'infezione (le specie del genere Prevotella producono proteasi che degradano le immunoglobuline) (3). L'infezione anaerobica può essere facilitata dalla somministrazione di antibiotici come i chinoloni o fluorochinoloni, trimethoprim-sulfametossazolo e aminoglicosidi a cui i batteri anaerobi sono notevolmente resistenti (6).

\section{DESCRIZIONE DEL CASO}

Paziente di 10 anni di età che accusa iperpiressia saltuaria da circa dieci giorni con notevole faringodinia. Dopo trattamento con cefixima (x $6 \mathrm{gg}$ ) e successiva terapia con piperacillina $\mathrm{x}$ i.m. (2 gr die x $3 \mathrm{gg}$ ) e bentelan x i.m. (1,5mg die x 3 giorni) è stato constatato un aggravamento della sintomatologia, con disfagia, alitosi e secrezione nasale mucopurulenta. Il paziente è stato pertanto ricoverato presso l'U.O. di Otorinolaringoiatria. In seguito alla visita otorinolaringoiatrica, è stata osservata una notevole ipertrofia dei pilastri ton- 
sillari e delle tonsille palatine che hanno mostrato un aspetto necrotico, tappezzate da materiale di colore grigiastro striato di sangue, fetido e adeso. È stata inoltre notata una tumefazione bilaterale, in sede latero-cervicale, a livello delle logge parotidee e in sede sovraclaveare, dolenti alla palpazione. Il paziente presentava una temperatura corporea di $39^{\circ} \mathrm{C}$.

E stato eseguito un prelievo ematico ed è stata instaurata una terapia antibiotica con piperacillina x i.m. (2 gr. die x 2 gg), tobramicina (100mg.die x $2 \mathrm{gg}$ ) e bentelan (1,5 mg.die x $2 \mathrm{gg}$ ). Gli esami ematochimici hanno rilevato: $\mathrm{GB}=13.000 / \mathrm{mmc}$ (39\% neutrofili, $43 \%$ linfociti, luc 10\%; lo striscio periferico ha evidenziato la presenza di elementi linfomonocitoidi), VES:44, PCR:36 mg/1, GOT:43 UI/1, GPT:49UI/1,GGT:80UI/1; gli anticorpi anti-VCA IgM e anti-VCA IgG e anti EBNA IgM sono risultati positivi. Nonostante il trattamento antibiotico le condizioni cliniche del paziente hanno subito un ulteriore peggioramento mostrando difficoltà di alimentazione - ed è stata pertanto richiesta una consulenza infettivologica. E stata eseguita l'ecografia del collo e addome che ha evidenziato la presenza di multipli linfonodi aumentati di volume in sede laterocervicale bilateralmente, a livello delle logge parotidee ed in sede sovraclaveare bilateralmente; fegato e milza infine sono risultati di dimensioni aumentate. Dopo consulenza infettivologica vengono somministrati $\mathrm{x}$ e.v. tobramicina $(100 \mathrm{mg}$ die $\mathrm{x} 2$ gg), piperacillina (2 gr.die x $2 \mathrm{gg}$ ) e bentelan (1,5 $\mathrm{mg}$ die $\mathrm{x} 2 \mathrm{gg}$ ). Viene prelevato del materiale necrotico tonsillare per l'esame colturale. Il materiale tonsillare di aspetto necrotico-poltaceo viene sottoposto all'esame batterioscopico diretto previa colorazione di Gram ed all'esame colturale. Dall'esame batterioscopico è stata evidenziata la presenza di granulociti neutrofili e bacilli Gramnegativi. L'esame colturale è stato eseguito utilizzando i seguenti terreni di coltura: agar-sangue, agar-MacConkey ed agar-cioccolato ed incubati in aerobiosi alla temperatura di $37^{\circ} \mathrm{C}$; agarSchaedler e Schaedler con kanamicina e vancomicina ed incubati in anaerobiosi alla temperatura di $37^{\circ} \mathrm{C}$. Dopo 48 ore di incubazione è stata osservata crescita sulle piastre incubate in anaerobiosi; sulle colonie viene eseguito il test della catalasi (che risulta negativo) ed il test della cefinase (che risulta positivo). Per quest'ultimo saggio è stato utilizzato un dischetto contenente una cefalosporina cromogena. L'identificazione biochimica è stata effettuata utilizzando la galleria Rapid-ID32ANA (bioMérieux). L'antibiogramma è stato eseguito mediante galleria ATBANAbioMérieux, che consente di saggiare la sensibilità agli antibiotici in terreno semisolido in condi- zioni simili a quelle delle tecniche di riferimento di agar-diluizione, secondo le raccomandazioni dell'NCCLS (8). L'identificazione biochimica ha dato come risultato: Prevotella denticola. Il ceppo è risultato resistente a: penicillina, cefoxitina, cefotetan, piperacillina, clindamicina e metronidazolo; è risultato sensibile a: piperacillina + tazobactam, ticarcillina-clavulanato, amoxicillina-clavulanato, imipenem e cloramfenicolo. È stata pertanto instaurata una nuova terapia antibiotica con piperacillina+tazobactam x i.m. (2 gr.die $\mathrm{x} 3$ giorni), amoxicillina-clavulanato $x$ os (625 mg.die $x$ $10 \mathrm{gg}$ ) e bentelan x i.m. (1,5 mg.die x $3 \mathrm{gg}$ ). Dopo alcune ore dalla somministrazione di piperacillina+tazobactam, è stato osservato un notevole miglioramento delle condizioni cliniche del paziente, che ha cominciato ad alimentarsi e, dopo qualche giorno, all'esame obiettivo, è stata osservata una diminuzione di volume dei linfonodi in sede latero-cervicale bilateralmente ed in sede sovraclaveare bilateralmente, fegato e milza nella norma.

\section{CONCLUSIONI}

La mononucleosi è una malattia infettiva, che nella maggior parte dei casi ha una prognosi favorevole. Non esiste trattamento specifico antivirale ed è consigliata la somministrazione di steroidi a basse dosi, per bloccare almeno in parte l'eccessiva reazione linfocitaria che caratterizza la malattia. Le sovrainfezioni batteriche in corso di mononucleosi sono frequenti e in questi casi è necessario somministrare una terapia antibiotica per evitare le complicanze batteriche. Nel caso clinico descritto, la necrosi tonsillare causata dal virus di Epstein Barr ha favorito la colonizzazione delle tonsille da parte del batterio anaerobio Prevotella denticola, saprofita del cavo orale. Il ceppo isolato è risultato produttore di $\beta$-lattamasi e l'antibiogramma ha mostrato resistenza alle penicilline (amoxicillina, ticarcillina, piperacillina e penicillina), e cefalosporine (cefoxitina e cefotetan). Le condizioni cliniche del paziente sono decisamente migliorate dopo somministrazione di antibiotici $\beta$-lattamici combinati con inibitori di $\beta$-lattamasi (piperacillina+tazobactam, amoxicillina-clavulanato). Numerosi studi hanno dimostrato la presenza di $\beta$-lattamasi in percentuale variabile (dal 65\% all'87\%) nei ceppi di Prevotella isolati dal cavo orale di bambini; la frequenza di specie resistenti era associata a precedenti esposizioni dei bambini agli antibiotici (9). È stato inoltre osservato che molte specie del genere Prevotella (tra cui Prevotella denticola) possiedono dei transposoni coniugativi che mediano il trasferimento di geni ad altri batteri dello stesso genere, e che sono portatori di fattori genetici per l'anti- 
biotico-resistenza, determinando la diffusione di ceppi resistenti (1).

\section{BIBLIOGRAFIA}

1. Arzese AR, Tomasetig L, Botta G. Detection of Teq and ermF antibiotic resistance genes in Prevotella and Porphyromonas isolates from clinical specimens and resident microbiota of humans. J Antimicrob Chemother 2000; 45: 577-82.

2. Bailey RE. Diagnosis and treatment of infectious mononucleosis. Am Fam Physician 1994; 49: 879-88.

3. Durden B. Virulence factors in anaerobes, Clin Infect Dis 1994; 18(suppl 4): S253-S259.

4. Englund JA. The many faces of Epstein-Barr virus, Postgrad Med 1988; 83: 167-79.

5. Finegold SM, Baron EJ, Wexler HM. A clinical guide to anaerobic infectious, Belmont, Calif, 1992-Star.

6. Jousimies-Somer H, Summonen P, Finegold S. Bacteroides, Porphyromonas, Prevotella, Fusobacterium and other anaerobic Gram-negative bacteria. In Murray P et al, editors: Manual of Clinical Microbiology, ed 6th, Washington, DC, 1995, ASM.

7. Mark H. EBELL, M.D. M.S. Epstein-Barr virus Infectious Mononucleosis, J Am Acad Family Physicians, 2004.

8. National Committee for Clinical Laboratory Standards. Methods for Antimicrobial susceptibility testing of Anaerobic bacteria. M11-A5, 2000.

9. Nyfors S, kononen E, Takala A, Jousimies-Somer H. $\beta$-lactamase production by oral anaerobic GramNegative species in infants in Relation to Previous. Antimicrobial Therapy. Antimicrob Agents Chemother, 1999; vol 43: 1591-4.

Maria Teresa Allù

Laboratorio di analisi chimico-cliniche e microbiologiche

Azienda Ospedaliera Ragusa

P.zza Ospedale Civile, 1

97100 Ragusa

E-mail: m.allu@tiscali.it 